\title{
Decoding the phosphorylation code in Hedgehog signal transduction
}

\author{
Yongbin Chen ${ }^{1}$, Jin Jiang ${ }^{2}$
}

${ }^{I}$ Key Laboratory of Animal Models and Human Disease Mechanisms of Chinese Academy of Sciences \& Yunnan Province, Kunming Institute of Zoology, Chinese Academy of Sciences, 32 Jiaochang Donglu, Kunming, Yunnan 650223, China, ${ }^{2}$ Department of Developmental Biology, University of Texas Southwestern Medical Center at Dallas, Dallas, TX 75390, USA

Hedgehog (Hh) signaling plays pivotal roles in embryonic development and adult tissue homeostasis, and its deregulation leads to numerous human disorders including cancer. Binding of Hh to Patched (Ptc), a twelve-transmembrane protein, alleviates its inhibition of Smoothened (Smo), a seven-transmembrane protein related to G-proteincoupled receptors (GPCRs), leading to Smo phosphorylation and activation. Smo acts through intracellular signaling complexes to convert the latent transcription factor Cubitus interruptus (Ci)/Gli from a truncated repressor to a fulllength activator, leading to derepression/activation of Hh target genes. Increasing evidence suggests that phosphorylation participates in almost every step in the signal relay from $\mathrm{Smo}$ to $\mathrm{Ci} / \mathrm{Gli}$, and that differential phosphorylation of several key pathway components may be crucial for translating the Hh morphogen gradient into graded pathway activities. In this review, we focus on the multifaceted roles that phosphorylation plays in Hh signal transduction, and discuss the conservation and difference between Drosophila and mammalian Hh signaling mechanisms.

Keywords: Hedgehog; Smo; Gli; signal transduction; phosphorylation; development; cancer

Cell Research (2013) 23:186-200. doi:10.1038/cr.2013.10; published online 22 January 2013

\section{Introduction}

The Hedgehog $(\mathrm{Hh})$ signaling pathway plays crucial roles in the control of cell growth and patterning during embryonic development and adult tissue homeostasis [1, 2]. Malfunction of this pathway has been linked to numerous human disorders including cancer $[1,3]$. hh was first identified as a segment polarity gene in Drosophila and the core pathway was delineated by fly geneticists $[2$, 4]. Vertebrates contain multiple $h h$ family members; e.g., mammals contain three $h h$ genes: Sonic Hedgehog (Shh), Indian Hedgehog (Ihh) and Desert Hedgehog (Dhh), with $S h h$ playing a prevalent role [3]. Hh proteins are dual lipid modified and form soluble protein complexes that promote its long-range signaling $[3,5,6]$. Secretion of lipidated $\mathrm{Hh}$ is mediated by a twelve-transmembrane protein Dispatched (Disp) as well as a glycoprotein

Correspondence: Yongbin Chen ${ }^{\mathrm{a}}$, Jin Jiang ${ }^{\mathrm{b}}$

${ }^{\mathrm{a}}$ Tel: +86-0871-5176312

E-mail: ybchen@mail.kiz.ac.cn

${ }^{\mathrm{b}} \mathrm{Tel}$ : +01-2146455914

E-mail: jin.jiang@utsouthwestern.edu
Scube/You (vertebrate only) [7-10], whereas Hh gradient formation is regulated by HSPGs and other cell surface molecules $[6,11]$.

The Hh signal is transduced by a conserved core signaling pathway that culminates in the activation of a latent $\mathrm{Zn}$-finger transcription factor Cubitus interruptus (Ci)/Gli (Figure 1). Vertebrates contain three Gli proteins: Gli1, Gli2 and Gli3, with Gli2/3 as the primary mediators of Hh signaling and Glil as a target of the $\mathrm{Hh}$ pathway that acts in a positive feedback to reinforce the Gli activity [12]. In the absence of $\mathrm{Hh}, \mathrm{Ci} / \mathrm{Gli}$ (mainly Gli3 and to a lesser extent Gli2) is proteolytically processed into a truncated form $\left(\mathrm{Ci}^{\mathrm{R}} / \mathrm{Gli}^{\mathrm{R}}\right)$ that functions as a transcriptional repressor to block the expression of a subset of Hh target genes. Hh signaling inhibits $\mathrm{Ci} / \mathrm{Gli}$ processing and thus the production of $\mathrm{Ci}^{\mathrm{R}} / \mathrm{Gli}^{\mathrm{R}}$, and converts the accumulated full-length $\mathrm{Ci} / \mathrm{Gli}\left(\mathrm{Ci}^{\mathrm{F}} / \mathrm{Gli}^{\mathrm{F}}\right)$ into an active form $\left(\mathrm{Ci}^{\mathrm{A}} / \mathrm{Gli}^{\mathrm{A}}\right)$ that stimulates Hh target gene expression.

\section{Hh signaling pathway: an overview}

The core components of the Hh pathway were initially 

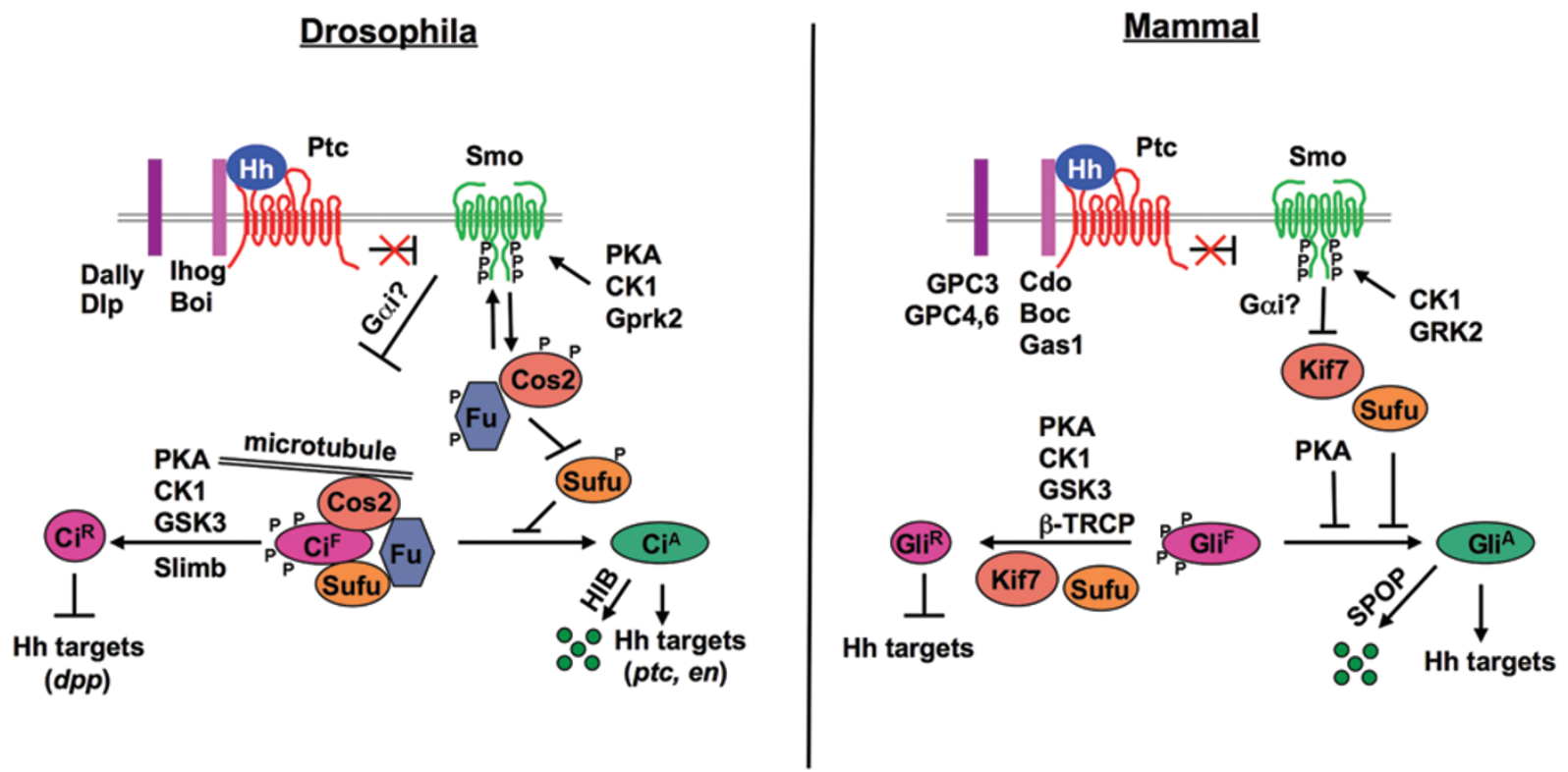

\begin{abstract}
Figure $1 \mathrm{Hh}$ signal transduction in Drosophila and mammalian systems. Hh protein is lipid-modified. Drosophila and mammalian HSPGs, Dally, Dally-like (DIp), GPC3, GPC4 and GPC6 modulate Hh signaling. In the absence of Hh, Ptc inhibits Smo, allowing $\mathrm{Ci}^{\mathrm{F}} / \mathrm{Gli}^{\mathrm{F}}$ to be phosphorylated by multiple kinases and targeted for Slimb/ $\beta$-TRCP-mediated proteolysis to generate $\mathrm{Ci}^{\mathrm{R}} / \mathrm{Gli}^{\mathrm{R}}$. In Drosophila, the kinesin-like protein Cos2 acts as a molecular scaffold to bridge Ci to its kinases. In the presence of Hh, Ptc inhibition of Smo is released, which triggers Smo phosphorylation by PKA, CK1 and Gprk2/GRK2, leading to its cell surface accumulation and activation. Smo recruits Cos2-Fu complex to the cell surface, and dissociates Cos2-Cikinase complexes to inhibit $\mathrm{Ci}$ phosphorylation and processing. In the presence of high levels of $\mathrm{Hh}, \mathrm{Fu}$ converts $\mathrm{Ci}^{\mathrm{F}}$ into $\mathrm{Ci}^{\mathrm{A}}$ by antagonizing Sufu inhibition. $\mathrm{Ci}^{\mathrm{A}}$ is unstable and degraded by the HIB-mediated Ub/proteasome pathway. Fu-Cos 2 also regulates Smo phosphorylation in a feedback loop mechanism. In mammals, Hh induces Smo phosphorylation by CK1 and GRK2, leading to its ciliary accumulation (not shown here) and activation. Sufu is a major whereas Kif7 a minor inhibitor of Gli proteins. The HIB homolog SPOP is responsible for degrading Gli proteins in the absence of Sufu. $\mathrm{Ci}^{\mathrm{F}} / \mathrm{Gli}^{\mathrm{F}}$ : full length $\mathrm{Ci} / \mathrm{Gli}$; $\mathrm{Ci}^{\mathrm{A}} / \mathrm{Gli}{ }^{\mathrm{A}}$ : activator form of $\mathrm{Ci} / \mathrm{Gli} ; \mathrm{Ci}^{\mathrm{R}} / \mathrm{Gli}$ : repressor form of $\mathrm{Ci} / \mathrm{Gli}$. Adapted from reference [1].
\end{abstract}

identified in Drosophila and are conserved in vertebrates. These include Patched (Ptc), a twelve-transmembrane protein that is structurally related to the bacterial RND family of proton-driven transporters [13], and Smoothened ( $\mathrm{Smo}$ ), a seven-transmembrane protein that is structurally related to GPCRs [14-16]. Ptc functions as the Hh receptor, whereas Smo functions as a signal transducer $[16,17]$. Unlike most signal transduction pathways where receptors function as positive regulators of the pathways and activate downstream signaling components upon ligand binding, Ptc acts as a negative regulator of the Hh pathway by inhibiting Smo in the absence of the ligand [18]. Binding of Hh to Ptc, which is facilitated by the Ihog/Cdo family of coreceptors [19], alleviates its inhibition of Smo, leading to Smo phosphorylation and activation [20] (Figure 1).

Several conserved pathway components function to link Smo to $\mathrm{Ci} / \mathrm{Gli}$, including the kinesin-like protein Costal2 (Cos2)/Kif7 and Sufu, as well as several Ser/ Thr kinases including PKA, GSK3 and CK1 (Figure 1).
In Drosophila, Cos2 forms a complex with the Ser/Thr kinase Fused $(\mathrm{Fu})$ and $\mathrm{Ci}$ to impede $\mathrm{Ci}$ nuclear translocation and promote $\mathrm{Ci}$ processing into $\mathrm{Ci}^{\mathrm{R}}$ [21-26]. Sufu also forms a complex with $\mathrm{Ci}$ to impede its nuclear translocation and block $\mathrm{Ci}^{\mathrm{A}}$ activity $[23,27,28]$. In the absence of Sufu, $\mathrm{Ci}$ is still converted into $\mathrm{Ci}^{\mathrm{R}}$, and $\mathrm{Ci}^{\mathrm{F}}$ is sequestered in the cytoplasm by $\operatorname{Cos} 2$ so that $S u f u$ mutation does not cause discernable activation of the Hh pathway [28]. In contrast, Sufu knockout (KO) in mice leads to ectopic Hh pathway activation similar to loss of Ptc [29, 30], suggesting that Sufu plays a major role in restricting Gli activity downstream of Smo in the mammalian system. One possible reason is that in the mammalian Hh pathway, Sufu is required not only for inhibiting $\mathrm{Gli}^{\mathrm{A}}$ formation but also for the production of $\mathrm{Gli}^{\mathrm{R}}$ [31].

In Drosophila, the Fu kinase converts $\mathrm{Ci}$ into an active but labile form $\left(\mathrm{Ci}^{\mathrm{A}}\right)$ that is degraded via a Cul3-based E3 ubiquitin ligase containing the BTB family protein HIB (also called Rdx) [32-34]. Interestingly, hib expression is induced by Hh signaling in both Drosophila 
embryos and imaginal discs, thus forming a negative feedback loop to attenuate Hh pathway activity [32, 33]. SPOP, which is the vertebrate homolog of HIB, may play an analogous role in fine-tuning Hh signaling by degrading Gli2/3 proteins $[30,35,36]$. Unexpectedly, the mammalian $\mathrm{Fu}$ homolog $(\mathrm{mFu})$ is not required for Hh signaling because $\mathrm{mFu}^{-/}$mice do not exhibit any phenotypes indicative of the Hh pathway activity defect $[37,38]$.

Another major difference between the mammalian and Drosophila pathways is that mammalian but not Drosophila Hh signaling depends on the primary cilium, a microtubule-based membrane protrusion and antennalike cellular structure [39]. Genetic screens in mice identified multiple intraflagellar transport proteins (IFTs) critical for appropriate Hh signaling [40, 41]. Major Hh signaling pathway components including Ptc, Smo, Kif7, Sufu, PKA and Gli proteins are present in the primary cilium with some exhibiting a dynamic pattern [30, 4246]. For example, Hh promotes ciliary exit of Ptc but ciliary accumulation of Smo $[42,44]$. Hh also stimulates the accumulation of Gli proteins at the cilium tip, a step likely reflecting $\mathrm{Gli}^{\mathrm{A}}$ formation. Disruption of primary cilia impedes the formation of both $\mathrm{Gli}^{\mathrm{R}}$ and $\mathrm{Gli}^{\mathrm{A}}[40,43$, 47]. Thus, the primary cilium may function as a signaling center to orchestrate the molecular events leading to Gli processing in the absence of $\mathrm{Hh}$ and Gli activation in response to $\mathrm{Hh}$, although the exact biochemical mechanisms remain poorly understood.

\section{Regulation of Ci/Gli proteolysis: a story of three kinases}

$\mathrm{Ci} / \mathrm{Gli}$ processing is responsible for the production of $\mathrm{Ci}^{\mathrm{R}} / \mathrm{Gli}^{\mathrm{R}}$ that plays important roles in preventing inappropriate Hh pathway activation in many developmental contexts, including Drosophila and vertebrate limb development $[1,12]$. Unraveling the mechanism underlying $\mathrm{Ci} /$ Gli processing has been facilitated by genetic studies in Drosophila that identified two key components in this process: PKA and an F-box protein Slimb/ $\beta$-TRCP that functions as a substrate recognition component of the SCF family of E3 ubiquitin ligases [48-51]. An important feature of the SCF family of E3s is that they only recognize substrates after the substrates are phosphorylated, thus providing a link between kinase-mediated phosphorylation and ubiquitin/proteasome-mediated proteolysis $[52,53]$. Indeed, PKA can directly phosphorylate multiple $\mathrm{Ser} / \mathrm{Thr}$ residues in the $\mathrm{C}$-terminal half of $\mathrm{Ci}$ (Figure 2 ), and mutating these PKA sites abolishes Ci processing $[54,55]$. However, phosphorylation of Ci by PKA alone does not confer recognition by Slimb, implying that additional phosphorylation events are required for $\mathrm{Ci}$ pro- cessing. Further genetic studies identified Shaggy (Sgg), the Drosophila GSK3, and CK1 as two kinases that act in conjunction with PKA to promote $\mathrm{Ci}$ processing [5658]. These three kinases phosphorylate Ci sequentially at three clusters of sites, with PKA serving as the priming kinase for GSK3 and CK1 (Figure 2), and these phosphorylation events create the docking sites for $\mathrm{SCF}^{\text {Slimb }}$ $[57,59]$. Strikingly, mutating any of the three phosphorylation clusters diminished the production of $\mathrm{Ci}^{\mathrm{R}}$ in vivo, suggesting that they act cooperatively to regulate $\mathrm{Ci}$ processing $[56,57]$. A more recent study suggested that an extended phosphorylation cluster primed by PKA sites 1 and 2 (SpTpYYGSp), which closely resembles the $\mathrm{Slimb} / \beta$-TRCP binding site consensus $\mathrm{DSpGX}_{2-4} \mathrm{Sp}$, provides the primary contact site for Slimb (Figure 2), and that multiple phosphorylation events may lead to the recruitment of two copies of $\mathrm{SCF}^{\text {Slimb }}$ complex that bind $\mathrm{Ci}$ simultaneously [60]. Thus, efficient Slimb binding and $\mathrm{Ci}$ processing require phosphorylation at multiple sites by three kinases, rendering $\mathrm{Ci}$ proteolysis very sensitive to $\mathrm{Hh}$ regulation. Indeed, low levels of Hh signaling are sufficient to block the production of $\mathrm{Ci}^{\mathrm{R}}$ [61].

Similar phosphorylation events mediated through PKA, GSK3 and CK1 regulate proteolysis of Gli2 and Gli3 by recruiting $\beta$-TRCP $[36,62-66]$. In contrast to Gli3 where $\beta$-TRCP-mediated proteolysis leads to partial degradation and therefore the production of $\mathrm{Gli}^{\mathrm{R}}$, Gli2 proteolysis often leads to complete degradation of the protein, consistent with the genetic studies suggesting that Gli3 is the major contributor of $\mathrm{Gli}^{\mathrm{R}}$ [12]. Taking advantage of this difference, Pan et al. [67] identified a processing determinant domain (PDD) that is responsible for the differentially regulated limited proteolysis of Gli2/3. Several sequence and structural motifs are also required for proper $\mathrm{Ci}$ processing $[68,69]$, and removal of a $\mathrm{Ci}$ processing motif renders complete degradation of Ci $[60]$.

A recent study demonstrated that PKA is not only required for Gli3 processing but also has a critical role in restricting the activator activity of Gli2 [45]. PKAdeficient mice exhibited phenotypes similar to $\mathrm{Ptc}^{-/-}$or $\mathrm{Sufu}^{-/-}$mice with a full-blown ectopic activation of the Hh pathway and ventralized neural tubes. Removal of Gli2 from PKA mutant mice suppressed the ectopic Hh pathway activation. Furthermore, Gli2 was accumulated at the tips of primary cilia in PKA-null MEF cells in the absence of upstream signal, consistent with its being constitutively active [45]. A previous study revealed that PKA also regulates both the activator and repressor forms of $\mathrm{Ci}$ because loss of PKA not only leads to ectopic expression of $d p p$, which is repressed by $\mathrm{Ci}^{\mathrm{R}}$, but also $p t c$, which is activated by $\mathrm{Ci}^{\mathrm{A}}$, whereas loss of Slimb 

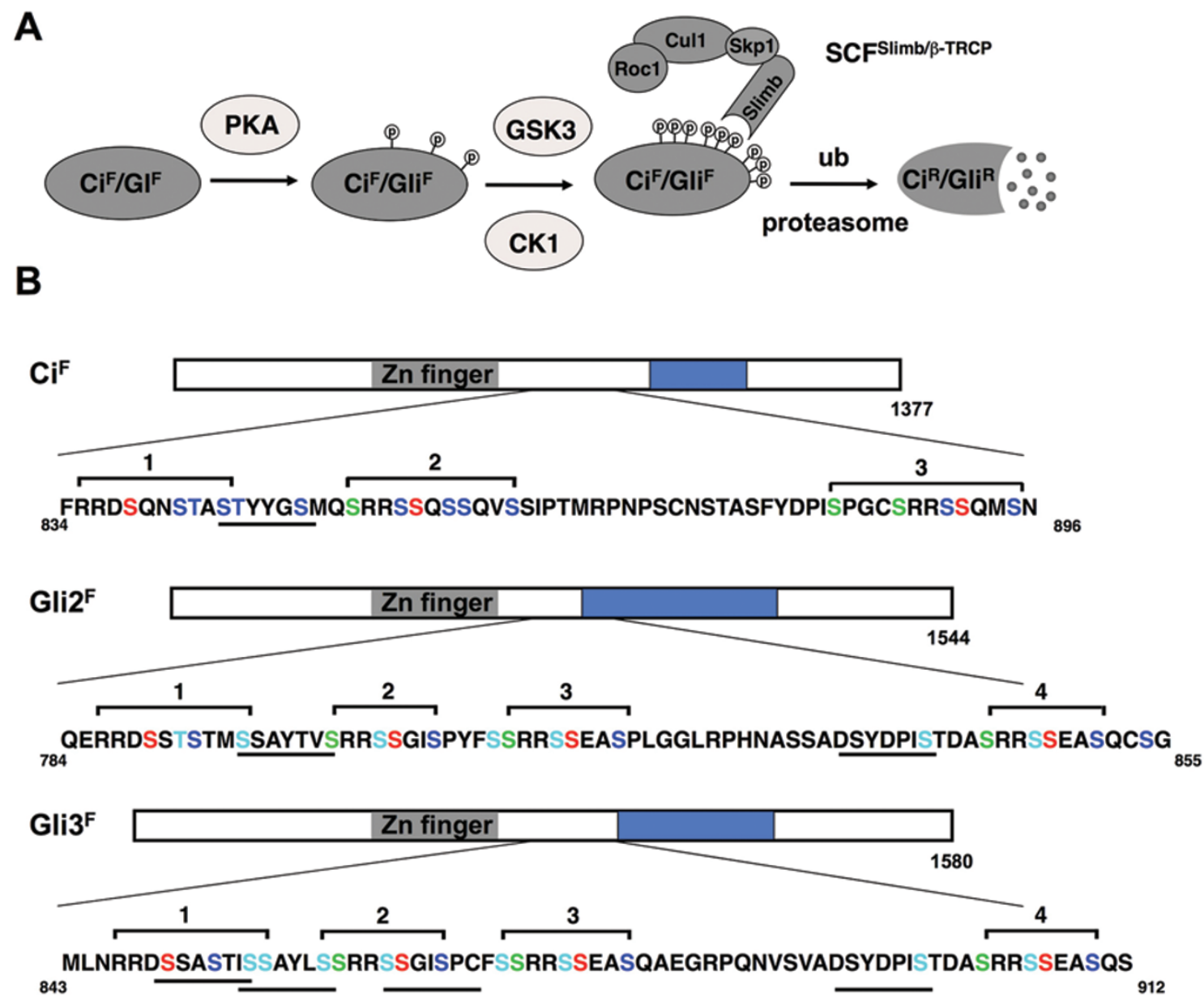

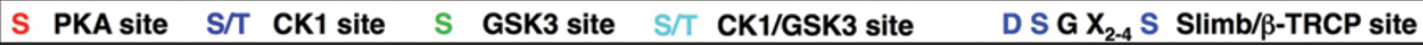

Figure $2 \mathrm{Ci} / \mathrm{Gli}$ phosphorylation and proteolysis. (A) In the absence of $\mathrm{Hh}, \mathrm{Ci}^{\mathrm{F}} / \mathrm{Gli}^{\mathrm{F}}$ is sequentially phosphorylated by $\mathrm{PKA}$, GSK3 and CK1, and targeted to Ub/proteasome-mediated proteolysis through Slimb/ $\beta$-TRCP to generate a truncated repressor $\mathrm{Ci}^{\mathrm{R}} / \mathrm{Gli}^{\mathrm{R}}$. (B) Diagrams of $\mathrm{Ci}$, mouse Gli2 and human Gli3 showing the PKA/GSK3/CK1 phosphorylation clusters in Ci/Gli and the Slimb/ $\beta$-TRCP binding consensus site. Putative Slimb/ $\beta$-TRCP binding sites in Ci/Gli are underlined. Grey and blue boxes denote $\mathrm{Zn}$-finger DNA binding domain and transactivation domain, respectively. ub: ubiquitin.

only leads to ectopic expression of $d p p$ but not ptc [49, 55, 68]. Furthermore, a proteolysis-resistant form of $\mathrm{Ci}$ is still inhibited by PKA [70], suggesting that PKA can restrict $\mathrm{Ci}^{\mathrm{A}}$ activity through a proteolysis-independent mechanism. It remains to be determined how PKA inhibits the activity of $\mathrm{Ci}^{\mathrm{A}} / \mathrm{Gli}^{\mathrm{A}}$.

GSK3, CK1 and Slimb/ $\beta$-TRCP are also involved in the Wingless(Wg)/Wnt pathway by regulating the proteolysis of $\beta$-catenin $[49,71,72]$, suggesting that these two developmental pathways employ a similar mechanism for signal transduction. These observations have also raised an important question of how pathway specificity is achieved. In the Wnt pathway, GSK3 and CK1 form a complex with $\beta$-catenin that is organized by the scaffold- ing proteins Axin and APC, and this processing complex is regulated by the specific interaction between the Wnt receptor complex and Axin [73]. An analogous mechanism has been proposed for the Drosophila Hh pathway in which Cos2 functions as a molecular scaffold to bridge $\mathrm{Ci}$ to its kinases, and $\mathrm{Hh}$ signaling impedes the formation of Cos2-Ci-kinase complexes [26]. Thus, Hh and Wnt may regulate distinct pools of GSK3 and CK1 to achieve pathway specificity.

In the vertebrate Hh pathway, primary cilia may have an important role in the regulation of Gli phosphorylation and proteolysis. For example, PKA is localized at the cilia base, suggesting that Gli proteins are phosphorylated during cilia transit [45]. This observation may 
explain why Gli processing is impeded in the absence of primary cilia. Both Sufu and Kif7 are required for Gli3 processing [30,31, 35, 74-77], raising a possibility that they may also regulate Gli3 phosphorylation. Indeed, Sufu can simultaneously bind GSK3 and Gli3, and thus recruit GSK3 to phosphorylate Gli3 [74]. Shh signaling may inhibit Gli phosphorylation by dissociating the Sufu-Gli-kinase complex [31, 46, 74].

\section{Smoothened phosphorylation code}

Smo belongs to the GPCR family and is an obligatory signal transducer of the canonical Hh signaling pathway. Oncogenic mutations in Smo contribute to basal cell carcinoma (BCC) and medulloblastoma [1, 78], and Smo has emerged as a prominent target for cancer therapeutics [79]. For example, vismodegib, a potent synthetic oral Smo inhibitor, has been approved by FDA for the treatment of advanced BCC. Thus, a complete understanding of how Smo is regulated and how it functions should not only provide important insights into the Hh signal transduction mechanisms but also may guide cancer drug development and treatment.

\section{Smo activation in flies: dual roles of PKA and CK1}

In Drosophila, $\mathrm{Hh}$ induces cell surface accumulation and phosphorylation of Smo [20]. Identification of Smo kinases came from the unexpected findings that PKA also plays a positive role in Hh signaling in both embryos and imaginal discs [80, 81]. Gain of PKA function promotes Smo accumulation and Hh pathway activation, whereas loss of PKA function blocks Hh-induced Smo accumulation as well as high levels of Hh signaling [81]. Similarly, inactivation of CK $1 \alpha / \varepsilon$ by RNAi blocks Hhinduced Smo accumulation and high levels of Hh signaling activity, suggesting that CK1 also plays dual roles in Hh signaling [81]. Interestingly, CK1 plays dual roles in Wnt/Wg signaling $[72,82]$, extending the similarity between these two pathways.

Biochemical studies demonstrated that PKA and CK1 sequentially phosphorylate three clusters of Ser/Thr residues in the Smo C-terminal cytoplasmic tail (C-tail), with PKA serving as the priming kinase for CK1 phosphorylation [81, 83, 84] (Figure 3A). Phospho-deficient Smo variants exhibit reduced cell surface expression and diminished Hh signaling activity, whereas phosphomimetic Smo variants exhibit increased cell surface levels and constitutive activity [81, 83, 84]. Interestingly, increasing the number of phospho-mimetic mutations in Smo results in a progressive elevation of Smo activity, suggesting that graded Hh signals may induce different levels of Smo activity through differential phosphoryla- tion [81]. Indeed, a recent study using a phospho-specific antibody to monitor Smo phosphorylation has provided evidence that Hh induces Smo phosphorylation in a dosedependent manner [85].

\section{Phospho-regulation of Smo trafficking and conformation}

Two recent studies suggested that phosphorylation promotes Smo cell surface expression by inhibiting ubiquitination-mediated endocytosis and degradation [86, 87]. In the absence of Hh, Smo is both mono- and polyubiquitinated at multiple Lys residues in its $\mathrm{C}$-tail, leading to its endocytosis and degradation by both lysosome- and proteasome-dependant mechanisms. Smo endocytosis is also promoted by Kurtz (Krz), the Drosophila non-visual arrestin $[87,88]$. Hh inhibits Smo ubiquitination and attenuates $\mathrm{Smo} / \mathrm{Krz}$ interaction, thereby stabilizing Smo on the cell surface [86, 87]. The E3 ligase(s) that catalyzes Smo ubiquitination has remained elusive but a deubiquitinating enzyme, UBPY/USP8, is required for Hh-induced deubiquitination of Smo [86, 87]. Interestingly, UBPY/ USP8 is also required for the deubiquitination of the Wnt receptor, $\mathrm{Fz}$ [89], thus providing another link between the Hh and Wnt pathways.

In addition to the regulation of Smo trafficking, phosphorylation also controls Smo conformation. Using FRET analysis, Zhao et al. [90] provided the first evidence that phosphorylation activates Smo by inducing a conformational switch of Smo C-tail, and it does so by antagonizing multiple Arg clusters in an autoinhibitory domain (SAID) located in the Smo C-tail (Figures $3 \mathrm{~A}$ and $3 \mathrm{~B}$ ). The Arg clusters maintain the Smo C-tail in a closed conformation through intramolecular electrostatic interaction; Hh-induced phosphorylation abrogates this interaction and promotes an open conformation and clustering of Smo C-tails (Figure 3B). An interesting feature of the SAID domain is that it contains multiple regulatory modules each of which consists of an Arg cluster linked to a phosphorylation cluster (Figure 3A). The pairing of positive and negative regulatory elements offers a more precise mode of regulation in response to graded Hh signals, as increasing levels of phosphorylation may gradually neutralize the negative effect of multi-Arg clusters, leading to a progressive change in Smo cell surface accumulation, conformation and activity [90]. Indeed, decreasing the number of functional Arg motifs has the same effect as increasing the number of phospho-mimetic mutations, both leading to a progressive increase of Smo activity [81,90].

\section{Regulation of Smo by additional kinases}

Phospho-mimetic mutations in the three PKA/CK1 phosphorylation clusters render Smo constitutively ac- 

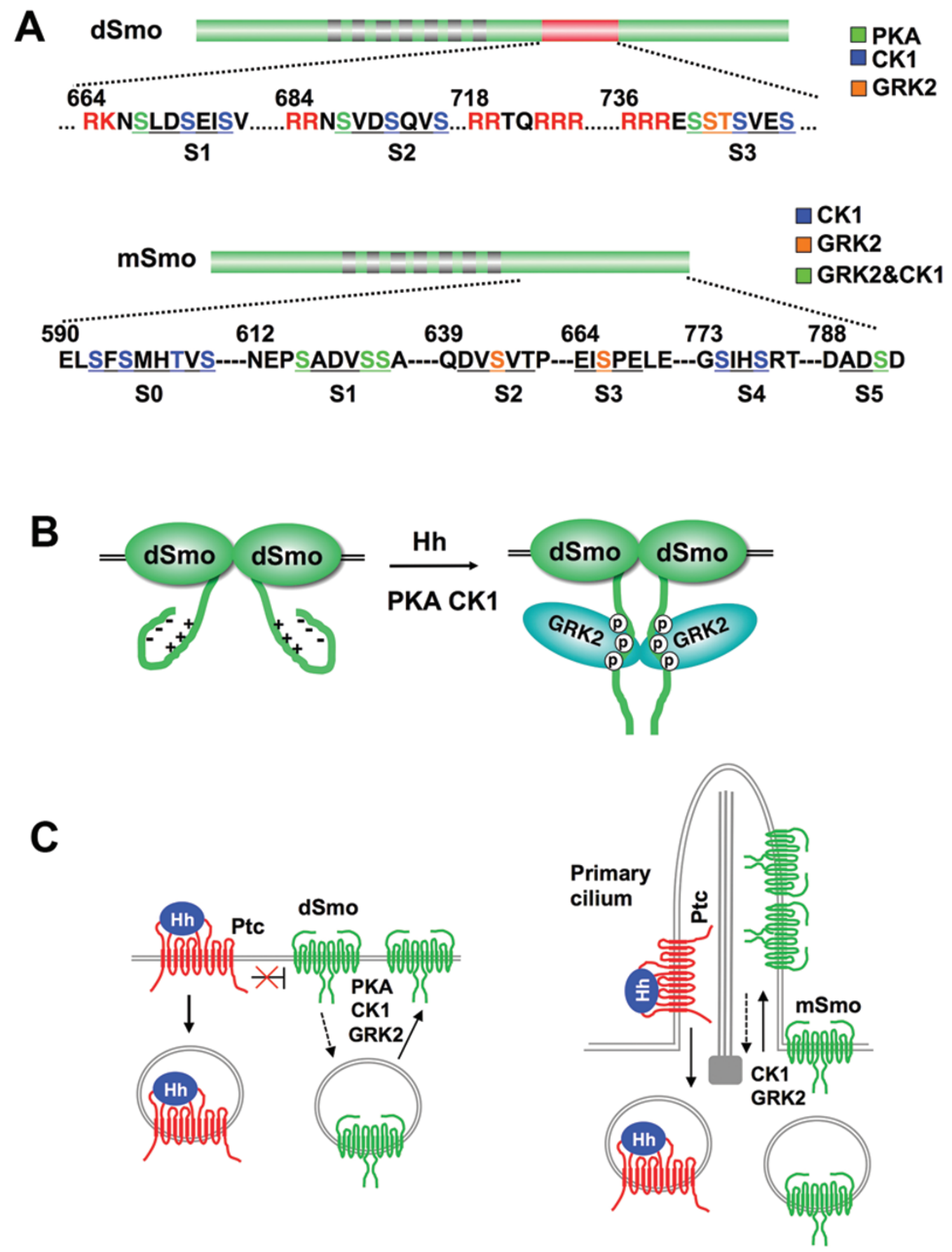

Figure 3 Phosphorylation of Smo regulates its subcellular localization and conformation in both Drosophila and mammalian systems. (A) Diagrams showing the phosphorylation sites in Drosophila (top) and mammalian Smo (bottom). The Arg motifs adjacent to phosphorylation clusters in Drosophila Smo are indicated in red. The red box in Drosophila Smo C-tail denotes the SAID domain. (B) Diagrams of closed inactive (left) and open active (right) conformations of Drosophila Smo. The closed conformation is maintained by intramolecular electrostatic interactions between multiple Arg motifs in the SAID domain and multiple acidic clusters in the C-terminal region of Smo. Hh-induced phosphorylation by PKA and CK1 neutralizes the positive charges and disrupts the intramolecular electrostatic interactions, leading to the opening and dimerization of Smo C-tail. Binding of GRK2 to Smo C-tail stabilizes its dimerization. (C) In Drosophila, Smo phosphorylation by PKA and CK1 leads to its cell surface accumulation and active conformation whereas in mammals, Smo phosphorylation by CK1 and GRK2 leads to its ciliary accumulation and active conformation. SAID: Smo autoinhibitory domain. Adapted from the references [1, 92, 100]. 
tive but fail to confer full activity [90], suggesting that full Smo activation may involve additional mechanisms. Such mechanisms may include additional phosphorylation events, non-phosphorylation events or both. Indeed, a mass spectrometry analysis identified many other phosphorylation sites in Smo C-tail, besides the three PKA/CK1 phosphorylation clusters [84]. Genetic modifier screens identified casein kinase 2 (CK2) and G-protein-coupled receptor kinase 2 (Gprk2/GRK2) as positive regulators of Hh signaling [91, 92]. Previous studies also revealed a positive role of GRK2 in the mammalian $\mathrm{Hh}$ pathway [93-95]. A recent study showed that inhibition of CK2 downregulated the $\mathrm{Hh} / \mathrm{Gli}$ signaling in human lung cancer cell lines, suggesting a conserved positive role of CK2 in the mammalian Hh pathway [96]. In Drosophila, CK 2 promotes Hh signaling by regulating the stability of both Smo and $\mathrm{Ci}$ [91]. In vitro kinase assay indicated that CK2 directly phosphorylated multiple Ser residues in the Smo C-tail [91], some of which were also phosphorylated in cultured cells in the presence of Hh [84].

In Drosophila, GRK2 promotes high-level Hh signaling through both kinase-dependent and kinase-independent mechanisms $[92,97]$. GRK2 phosphorylates Smo C-terminal tail at Ser741/Thr742, which is facilitated by PKA/CK1-mediated phosphorylation at adjacent Ser residues [92] (Figure 3A). In addition, GRK2 forms a dimer and binds Smo to stabilize its open conformation, and promote the dimerization of Smo C-tail [92] (Figure 3B). Interestingly, GRK2 expression is induced by $\mathrm{Hh}$ signaling in wing imaginal discs, thus forming a positive feedback loop to facilitate high-level Hh signaling [92, 97].

\section{A conserved Smo activation mechanism in mammals}

Mammalian Smo (mSmo) diverges significantly from Drosophila Smo (dSmo) in the primary sequence of its $\mathrm{C}$-tail and does not contain the three PKA/CK1 phosphorylation clusters found in dSmo C-tail (Figure 3A). In addition, $\mathrm{mSmo}$ traffics through the primary cilia to transduce the Hh signal (Figure 3C). These and other differences have led to the proposal that $\mathrm{mSmo}$ and $\mathrm{dSmo}$ are regulated by fundamentally distinct mechanisms [98, 99]. However, a recent study has revealed unexpected similarities between $\mathrm{dSmo}$ and $\mathrm{mSmo}$ activation mechanisms: both $\mathrm{dSmo}$ and $\mathrm{mSmo}$ are regulated by multi-site phosphorylation in a dose-dependent manner, and phosphorylation regulates both their subcellular localization and conformation [100] (Figure 3C).

Although PKA is not involved in mSmo activation, $\mathrm{CK} 1 \alpha$ and GRK2 are required for Hh-induced $\mathrm{mSmo}$ phosphorylation and pathway activation $[93,94,100$,
101]. CK1 $\alpha$ and GRK2 bind $\mathrm{mSmo}$ in response to $\mathrm{Hh}$ stimulation and phosphorylate $\mathrm{mSmo} \mathrm{C}$-tail at six Ser/ Thr clusters (S0-S5; Figure 3A) [100]. Functional study using both cultured mammalian cells and chick neural tubes suggested that multiple CK1/GRK2 phosphorylation sites regulate $\mathrm{mSmo}$ activity in a dose-dependent manner, with the two membrane-proximal clusters (S0 and S1) playing a major role [100]. As is the case for $\mathrm{dSmo}[81,85], \mathrm{mSmo}$ phosphorylation is regulated in a dose-dependent manner with increasing amounts of $\mathrm{Hh}$ inducing a progressive increase of $\mathrm{mSmo}$ phosphorylation, suggesting that $\mathrm{Hh}$ gradient is translated into an mSmo phosphorylation and activity gradient [100].

A prevalent view is that $\mathrm{Hh}$ activates $\mathrm{mSmo}$ by inducing its ciliary localization [42, 44] (Figure 3C). Indeed, CK1/GRK2-mediated phosphorylation promotes mSmo ciliary localization by recruiting $\beta$-arrestins that link $\mathrm{mSmo}$ to the anterograde kinesin-II motor $[100,102]$. However, ciliary localization of $\mathrm{mSmo}$ is insufficient for its activation because the Hh pathway inhibitor cyclopamine promotes rather than blocks ciliary localization of $\mathrm{mSmo}$ [103-105]. In addition to regulating $\mathrm{mSmo}$ ciliary localization, Hh induces a conformational switch that results in the dimerization/oligomerization of $\mathrm{mSmo}$ C-tail [90]. A similar conformational change is also induced by oncogenic Smo mutation (A1/M2) and Smo agonist SAG but blocked by cyclopamine, suggesting that Hh-induced mSmo conformational switch represents an additional and critical step for mSmo activation [90]. Hh-induced conformational switch is governed by CK1/ GRK2-mediated multi-site phosphorylation of $\mathrm{mSmo} \mathrm{C-}$ tail [100]. Cyclopamine traps ciliary-localized mSmo in an unphosphorylated form that adopts an inactive conformation, whereas ciliary-localized $\mathrm{mSmo}$ in response to $\mathrm{Hh}$ or Smo agonists is phosphorylated and thus adopts an active conformation [100]. Thus, Smo phosphorylation is a more faithful readout for pathway activation than Smo ciliary localization, and can serve as a biomarker for cancers caused by deregulated Hh pathway activation.

Hh stimulates the binding of both CK $1 \alpha$ and GRK2 to $\mathrm{mSmo}$, which likely contributes to the signal-induced mSmo phosphorylation [100]. In the absence of $\mathrm{Hh}$, the kinase-binding pockets in $\mathrm{mSmo}$ are masked when mSmo C-tail adopts a closed conformation. Shh stimulates $\mathrm{CK} 1 \alpha$ binding to a juxtamembrane site, likely by inducing a local conformational change, to initiate the phosphorylation and conformational change of $\mathrm{mSmo}$ C-tail. This further increases the binding of CK1 $\alpha /$ GRK2 to $\mathrm{mSmo} \mathrm{C}$-tail, forming a positive feedback loop to increase or sustain mSmo phosphorylation [100]. Interestingly, CK $1 \alpha$ is accumulated in the primary cilia in response to Hh stimulation, which may explain, at least in 
part, why phosphorylation of $\mathrm{mSmo}$ is more effective in primary cilia [100].

\section{Hh signal transduction: from Smo to Ci/Gli}

Smo can function as a GPCR to directly activate Gai in frog melanophores as well as in Drosophila and mammalian cultured cells [106-108]. In Drosophila wing discs, Gai is required for the expression of Hh target gene $d p p$ [108]; however, a physiological role for Gai in Shh signaling has not been demonstrated [109]. On the other hand, Smo can act via a G-protein-independent mechanism to relay the Hh signal to downstream signaling components $[1,110]$. In addition, downstream signaling components, including $\mathrm{Cos} 2, \mathrm{Fu}$, Sufu and perhaps $\mathrm{Ci} / \mathrm{Gli}$, are phosphorylated in response to Hh stimulation, although the kinases involved and the physiological relevance of these phosphorylation events are not fully understood.

\section{Cos 2 phosphorylation and regulation of Hh signaling complexes}

Several studies demonstrated that Smo interacts with a Cos2-Fu complex through its C-tail [108, 111-115]. The interaction between $\mathrm{Smo}$ and $\mathrm{Cos} 2 / \mathrm{Fu}$ can occur even in the absence of $\mathrm{Hh}$ but there is evidence that Smo-Cos2$\mathrm{Fu}$ complex formation is facilitated by $\mathrm{Hh}$ signaling in both Drosophila embryos and cultured cells [114, 116, 117]. How the dynamic interaction between Smo and Cos2-Fu complex is regulated remains poorly understood, but it is possible that Smo and Cos2-Fu form distinct complexes depending on the phosphorylation status of individual components, and pathway activation may rely on changes in the location, composition and conformation of the complexes. Indeed, deletion analyses identified two distinct regions of Smo C-tail capable of binding to $\operatorname{Cos} 2$ : a membrane proximal domain between aa 651-686 and a C-terminal domain between aa 8181035 [111, 112]. The membrane proximal domain appears to mediate an inhibition of Smo phosphorylation by Cos 2 that forms a complex with PP4, and Cos2/PP4mediated inhibition of Smo phosphorylation is alleviated by $\mathrm{Fu}$ upon $\mathrm{Hh}$ signaling through a positive feedback loop [118-120]. In contrast, the C-terminal Cos2-binding domain plays a positive role in Smo activity [111], and its association with $\operatorname{Cos} 2$ is stimulated by $\mathrm{Hh}[116,117]$.

The interaction between Cos2 and different Smo domains appears to be regulated by $\operatorname{Cos} 2$ phosphorylation. In response to $\mathrm{Hh}, \mathrm{Fu}$ phosphorylates Cos 2 at Ser572 and Ser931 [121-123]. Using phospho-specific antibodies that recognize pSer572 or pSer931, Ranier et al. [123] provided evidence that Ser572 phosphorylation can be induced by low levels of Hh, whereas Ser931 phosphorylation only occurs in the presence of high levels of $\mathrm{Hh}$. In addition, they showed that Ser931 phosphorylation correlated with increased $\mathrm{Smo} / \mathrm{Cos} 2$ association and $\mathrm{pS} 931$ signal was mainly detected at the plasma membrane. Previous studies showed that Ser572 phosphorylation attenuated the association between Cos 2 and Smo, and that a phospho-mimetic form of Cos2 (Cos2S572D) exhibited reduced binding to the membrane proximal domain of Smo C-tail [119, 122]. Taken together, these results suggest that differential phosphorylation of Cos2 may regulate the formation of distinct Smo-Cos2-Fu complexes: Ser572 phosphorylation disrupts the inactive complex in which Cos2 binds to the membrane proximal region of Smo C-tail, whereas Ser931 phosphorylation favors the formation of an active complex in which Cos2 binds to the C-terminal region of Smo. However, whether Ser931 phosphorylation is the cause or consequence of stable Smo-Cos2-Fu complex formation has not been firmly established.

\section{Fu phosphorylation and action}

The Ser/Thr kinase $\mathrm{Fu}$ is a positive regulator of $\mathrm{Hh}$ signaling in Drosophila as well as in zebrafish [124, 125]. Fu contains an amino-terminal catalytic domain $(\mathrm{Fu}-\mathrm{KD})$ and a $\mathrm{C}$-terminal regulatory domain $(\mathrm{Fu}-\mathrm{RD})$ that mediates $\mathrm{Fu}$ interaction with other proteins including Cos2 and Sufu $[22,126]$. In addition, Fu-RD can interact with $\mathrm{Fu}-\mathrm{KD}$, and this intramolecular interaction may provide a mechanism for $\mathrm{Fu}$ autoinhibition [127]. In response to $\mathrm{Hh}$ or activated $\mathrm{Smo}, \mathrm{Fu}$ is hyperphosphorylated in a manner dependant on Fu kinase activity and CK1 [112, 116, 128, 129], suggesting that signal-stimulated Fu phosphorylation may involve both autophosphorylation and phosphorylation by other kinases. Many kinases can be activated by dimerization-induced transphosphorylation of their kinase domains within the activation loops [130]. Indeed, FRET analyses demonstrated that $\mathrm{Hh}$ induces dimerization of the $\mathrm{Fu}$ kinase domain in a dose-dependent manner $[116,117]$. Fu dimerization is mediated by a conformational switch and dimerization of the Smo C-tail, and depends on its association with Cos2 $[116,117]$ (Figure 4). Importantly, forced dimerization of $\mathrm{Fu}$ via heterologous dimerization domains can induce its autophosphorylation and $\mathrm{Hh}$ pathway activation [116, 117]. The requirement of Cos 2 for $\mathrm{Fu}$ dimerization and activation may explain the positive role of Cos 2 in $\mathrm{Hh}$ signaling observed previously [23, 24]. Indeed, expression of a dimerized $\mathrm{Fu}$ variant can restore high levels of Hh signaling in $\cos 2$ mutants [116].

The activation loop (AL) of Fu-KD contains four conserved Ser/Thr residues: Thr151, Thr154, Thr158 and 


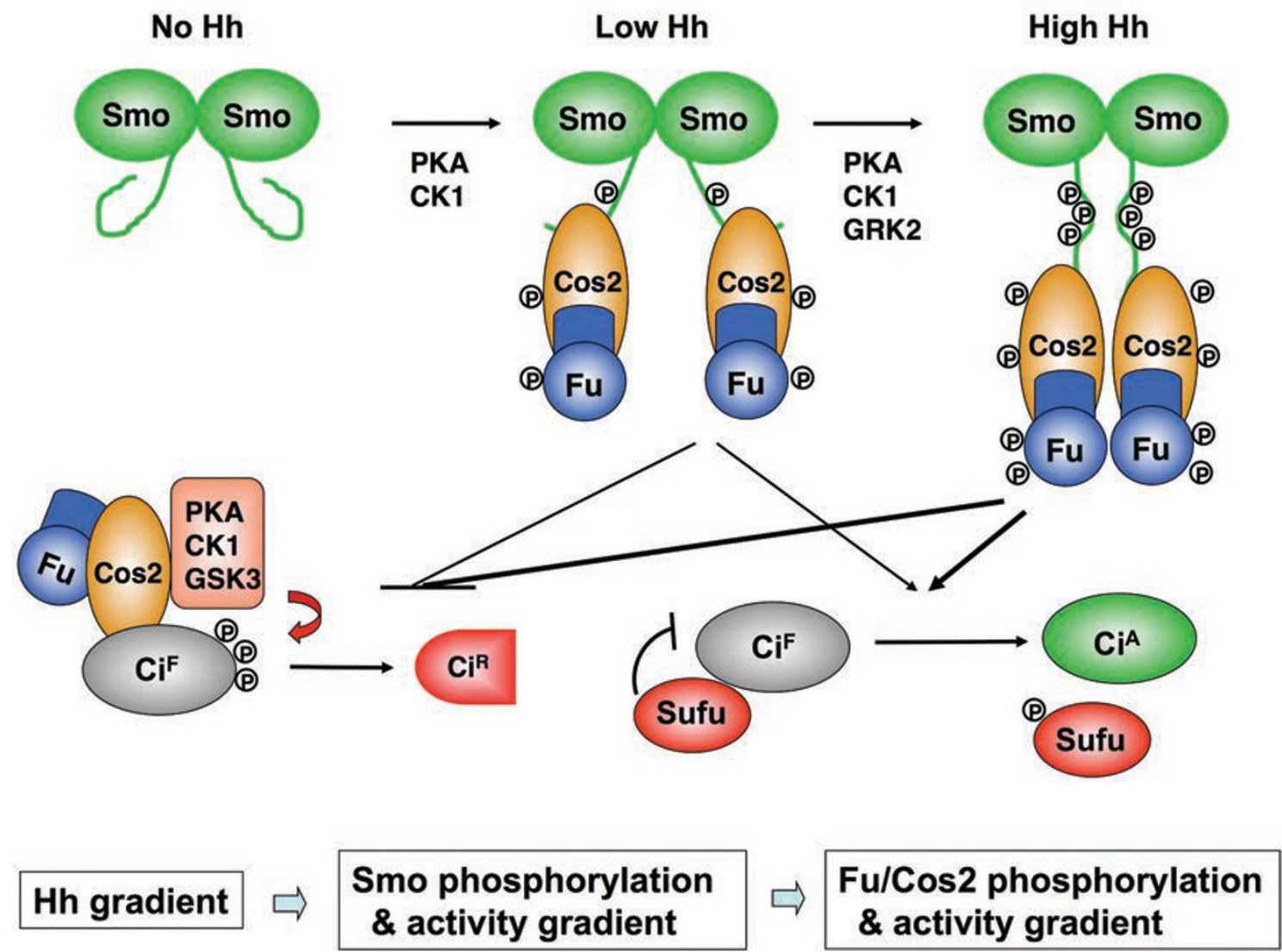

Figure $4 \mathrm{~A}$ model for Smo phosphorylation and conformational switch leading to Hh pathway activation in Drosophila. Hh morphogen gradient is translated into Smo phosphorylation and activity gradients, which are in turn translated into Fu/Cos2 phosphorylation and activity gradients. Smo phosphorylation induces an open conformation of Smo C-tail, which facilitates the recruitment of Cos2/Fu. In addition, dimerization of Smo C-tail leads to clustering of the bound Cos $2 / \mathrm{Fu}$, resulting in Fu phosphorylation and activation. Activated $\mathrm{Fu}$ inhibits $\mathrm{Ci}^{\mathrm{R}}$ production by dissociating Cos2-Ci-kinase complexes and stimulates $\mathrm{Ci}^{\mathrm{A}}$ formation likely by dissociating Sufu from $\mathrm{Ci}$. Adapted from the reference [116].

Ser159 [116, 129]. Mutagenesis experiments indicated that Ser159 is absolutely essential for Fu activity, whereas phosphorylation at the Thr residues is required for optimal activity; overall, there appears to be a correlation between the level of AL phosphorylation and the level of Fu activity $[116,129]$. Using phospho-specific antibodies that recognize dual phospho-residues, pThr151/pThr154 or pThr158/pSer159, Shi et al. [116] provided evidence that phosphorylation of these two clusters increased progressively in response to increasing levels of $\mathrm{Hh}$ or Smo phosphorylation, suggesting that $\mathrm{Hh} / \mathrm{Smo}$ activity gradient is translated into a $\mathrm{Fu}$ phosphorylation and activity gradient (Figure 4).

Fu-RD is phosphorylated both by Fu-KD and CK1, and these phosphorylation events may also regulate Fu kinase activity [116, 129]. Indeed, mutating several CK1 consensus sites in the Fu regulatory domain (such as Ser485 and Thr486) reduces the activity of a consti- tutively active form of $\mathrm{Fu}$ [129]. It is possible that CK1 may phosphorylate additional sites in the Fu-RD or even in the $\mathrm{Fu}-\mathrm{KD}$ to regulate $\mathrm{Fu}$ activity. It will be important to determine how phosphorylation of the Fu-RD contributes to Fu activation. One possibility is that phosphorylation of the Fu-RD may attenuate Fu autoinhibition.

A conventional view regarding its role in Hh signaling is that $\mathrm{Fu}$ is only required for transducing high levels of Hh signaling by converting $\mathrm{Ci}^{\mathrm{F}}$ into $\mathrm{Ci}^{\mathrm{A}}[28,124]$; however, the full spectrum of $\mathrm{Fu}$ function could have been underestimated due to the lack of a null mutation [131], and/or the existence of paralleled mechanisms such as Gai activation [108]. Using a gain-of-function approach, two recent studies demonstrated that activated forms of Fu can block $\mathrm{Ci}$ processing into $\mathrm{Ci}^{\mathrm{R}}$ in addition to converting $\mathrm{Ci}^{\mathrm{F}}$ into $\mathrm{Ci}^{\mathrm{A}}[116,129]$. Furthermore, overexpression of a dominant negative form of Fu blocks $\mathrm{Ci}$ accumulation and $d p p$ expression [131]. These new findings 
suggest that $\mathrm{Fu}$ may participate in the regulation of both $\mathrm{Ci}^{\mathrm{R}}$ and $\mathrm{Ci}^{\mathrm{A}}$. Mechanistically, activated $\mathrm{Fu}$ can phosphorylate $\operatorname{Cos} 2$, leading to the disassembly of the Cos2-Cikinase complexes required for $\mathrm{Ci}$ phosphorylation and processing (Figure 4) [116, 122, 123, 129]. Activated forms of Fu can also phosphorylate Sufu and dissociate Sufu from Ci, leading to $\mathrm{Ci}$ activation $[116,117]$ (Figure 4).

\section{Signaling downstream of mammalian Smo}

In mice, Sufu is a major negative regulator of Shh, signaling downstream of Smo [29, 132]. Sufu forms complexes with Gli proteins to inhibit their nuclear localization and transcriptional activity [133-136]. Recent studies using mammalian cultured cells revealed that Shh signaling induces dissociation of full-length Gli proteins from Sufu [31, 46], suggesting that inhibition of Sufu-Ci/ Gli complex formation could be a conserved mechanism for $\mathrm{Ci} / \mathrm{Gli}$ activation. Shh induces phosphorylation of full-length Gli3 that correlates with its nuclear localization and activation [31]. Although mammalian $\mathrm{Fu}$ is not required for Shh signaling, several kinases have been implicated in the regulation of Gli activity. For example, a kinome siRNA screen identified Cdc211 as a positive regulator of Shh signaling that acts downstream of Smo and upstream of Gli proteins [101]. Interestingly, Cdc211 can associate with Sufu to antagonize its repression of Gli in cultured cells and activate Shh pathway when overexpressed in zebrafish [101]. Furthermore, a Fu-related kinase, Ulk3, can phosphorylate Gli proteins in vitro and promote Gli1 transcriptional activities in cultured cells [137].

In addition to regulating Sufu/Gli association, Shh can also regulate Sufu stability through the ubiquitin/proteasome pathway in certain contexts, and thus indirectly regulates Sufu/Gli complex formation [138]. Interestingly, Sufu stability is regulated via dual phosphorylation at Ser342/Ser346 by PKA and GSK3, and blocking Sufu phosphorylation either by mutating Ser346 to Ala or by treating cultured cells with PKA inhibitors attenuates Sufu ciliary accumulation, whereas phospho-mimetic forms of Sufu exhibits increased ciliary localization [139]. The requirement of PKA-mediated phosphorylation for Sufu stabilization may account, at least in part, for the constitutive activation of Gli in PKA mutants [45].

As is the case for Cos2 in Drosophila Hh signaling, Kif7 also plays both positive and negative roles in mammalian Hh signaling [75-77]. A recent study suggested that Kif7 positively regulates $\mathrm{Hh}$ signaling in chondrocytes by destabilizing Sufu and restricting its ciliary accumulation [140]. In chondrocytes, Kif7 also inhibits Gli transcriptional activity independent of Sufu [77, 140].
Interestingly, a recent study provided evidence that Kif7 can form a complex with activated forms of Smo, and that the association requires the presence of two ciliary proteins, Evc and Evc2 [141], the products of genes responsible for the human disease Ellis-van Creveld syndrome $[142,143] . E v c^{-/-}$mice exhibit bone phenotypes indicative of Ihh signaling defects that mimic the human syndrome [142]. In cultured cells, Evc/Evc2 acts downstream of Smo but upstream of Gli and Sufu [141, 144]. Interestingly, Hh stimulates the binding of Evc/Evc2 to Smo depending on phosphorylation of the Smo C-tail and the primary cilia, suggesting that Evc/Evc2 may link activated Smo to downstream signaling components in the primary cilia [141, 144].

\section{Conclusion and future prospect}

Studies in the past decade have identified many kinases and numerous phosphorylation events that regulate Hh signal transduction. Biochemical and functional analyses of these kinases and phosphorylation events have provided critical insights into the Hh signal transduction mechanisms, and begun to address important biological questions such as how different levels of Hh signal are transduced. Given the power of new development in technologies such as mass spectrometry and genomewide in vivo RNAi screen, it is conceivable that additional kinases and phosphorylation events will be uncovered. Despite these achievements, insights into the regulation of many key phosphorylation events are still lacking. The opposing roles of PKA in the regulation of Smo and Ci have posed a unique challenge for understanding how phosphorylation by PKA is regulated. Hh signaling could in principle downregulate PKA through a Smo-GaicAMP axis [108]; however, direct evidence for a PKA activity change in response to $\mathrm{Hh}$ is still lacking [50]. Furthermore, downregulation of PKA does not explain how Hh stimulates Smo phosphorylation. Nevertheless, the involvement of PKA in Hh signaling does allow the pathway activity to be regulated by other GPCRs, as has been implicated by several recent studies $[145,146]$. The question of how Smo phosphorylation is regulated is intimately linked to the question of how Ptc inhibits Smo. A prevalent view is that Ptc functions as a transporter, regulating the subcellular distribution of lipophilic small molecules that act as either Smo agonists or antagonists [147]. Promising candidates for endogenous Smo agonists include oxysterols that influence Hh signaling by directly binding to Smo and promoting Smo phosphorylation $[100,148]$. It remains to be determined whether Ptc regulates oxysterols, and how binding of oxysterols to Smo influences its phosphorylation and activity. A re- 
cent study in Drosophila suggests that the phospholipid PI4P acts downstream of Ptc to regulate Smo cell surface expression and activity [149]. It awaits to be determined whether PI4P acts at a step upstream or downstream of Smo phosphorylation. At the other end of the pathway, the full spectrum of $\mathrm{Ci} / \mathrm{Gli}$ phosphorylation has not been explored. Although PKA-mediated phosphorylation of $\mathrm{Ci} /$ Gli inhibits Hh signaling, Ci/Gli may undergo other phosphorylation events resulting in pathway activation. In this regard, it is interesting to note that other pathways such as the mTOR/S6K pathway can promote $\mathrm{Hh}$ pathway activity by phosphorylating Gli1 in cancer cells [150]. One important future avenue would be to determine whether phosphorylation of $\mathrm{Ci} / \mathrm{Gli}$ is responsible for the conversion of $\mathrm{Ci}^{\mathrm{F}} / \mathrm{Gli}^{\mathrm{F}}$ into $\mathrm{Ci}^{\mathrm{A}} / \mathrm{Gli}^{\mathrm{A}}$ in response to Hh stimulation.

\section{Acknowledgments}

We apologize to those whose work we could not cite due to space limitations. Y Chen is supported by grants from the National Natural Science Foundation of China (31271579), National Key Basic Research Program of China (2013CB910900), American Heart Association postdoctoral fellowship (10POST3640046), Kunming Institute of Zoology startup foundation and Chinese Academy of Sciences. J Jiang is supported by grants from NIH (GM067045 and GM061269), CPRIT (RP100561) and Welch foundation (I-1603), and is a Eugene McDermott Endowed Scholar of Biomedical Science at UTSW.

\section{References}

1 Jiang J, Hui CC. Hedgehog signaling in development and cancer. Dev Cell 2008; 15:801-812.

2 Ingham PW, McMahon AP. Hedgehog signaling in animal development: paradigms and principles. Genes Dev 2001; 15:3059-3087.

3 Varjosalo M, Taipale J. Hedgehog: functions and mechanisms. Genes Dev 2008; 22:2454-2472.

4 Nusslein-Volhard C, Wieschaus E. Mutations affecting segment number and polarity in Drosophila. Nature 1980; 287:795-801.

5 Zeng X, Goetz JA, Suber LM, Scott WJ, Jr, Schreiner CM, Robbins DJ. A freely diffusible form of Sonic hedgehog mediates long-range signalling. Nature 2001; 411:716-720.

6 Guerrero I, Chiang C. A conserved mechanism of Hedgehog gradient formation by lipid modifications. Trends Cell Biol 2007; 17:1-5.

7 Amanai K, Jiang J. Distinct roles of central missing and dispatched in sending the Hedgehog signal. Development 2001; 128:5119-5127.

8 Burke R, Nellen D, Bellotto M, et al. Dispatched, a novel sterol-sensing domain protein dedicated to the release of cholesterol-modified hedgehog from signaling cells. Cell 1999; 99:803-815.

9 Ma Y, Erkner A, Gong R, et al. Hedgehog-mediated pat- terning of the mammalian embryo requires transporter-like function of dispatched. Cell 2002; 111: 63-75.

10 Creanga A, Glenn TD, Mann RK, Saunders AM, Talbot WS, Beachy PA. Scube/You activity mediates release of dually lipid-modified Hedgehog signal in soluble form. Genes Dev 2012; 26:1312-1325.

11 Yan D, Lin X. Shaping morphogen gradients by proteoglycans. Cold Spring HarbPerspect Biol2009; 1:a002493.

12 Hui CC, Angers S. Gli proteins in development and disease. Annu Rev Cell Dev Biol 2011; 27:513-537.

13 Hooper JE, Scott MP. The Drosophila patched gene encodes a putative membrane protein required for segmental patterning. Cell 1989; 59:751-765.

14 Alcedo J, Ayzenzon M, Von Ohlen T, Noll M, Hooper JE. The Drosophila smoothened gene encodes a seven-pass membrane protein, a putative receptor for the Hedgehog signal. Cell 1996; 86:221-232.

15 van-den-Heuval M, Ingham PW. Smoothened encodes a receptor-like serpentine protein required for hedgehog signalling. Nature 1996; 382:547-551.

16 Stone DM, Hynes M, Armanini M, et al. The tumour-suppressor gene Patched encodes a candidate receptor for Sonic hedgehog. Nature 1996; 384:129-134.

17 Chen Y, Struhl G. Dual roles for Patched in sequestering and transducing Hedgehog. Cell 1996; 87:553-563.

18 Ingham PW, Taylor AM, Nakano Y. Role of the Drosophila patched gene in positional signalling. Nature 1991; 353:184187.

19 Zheng X, Mann RK, Sever N, Beachy PA. Genetic and biochemical definition of the Hedgehog receptor. Genes Dev 2010; 24:57-71.

20 Denef N, Neubuser D, Perez L, Cohen SM. Hedgehog induces opposite changes in turnover and subcellular localization of patched and smoothened. Cell 2000; 102:521-531.

21 Sisson JC, Ho KS, Suyama K, Scott MP. Costal2, a novel kinesin-related protein in the Hedgehog signaling pathway. Cell 1997; 90:235-245.

22 Robbins DJ, Nybakken KE, Kobayashi R, Sisson JC, Bishop JM, Therond PP. Hedgehog elicits signal transduction by means of a large complex containing the kinesin-related protein costal2. Cell 1997; 90:225-234.

23 Wang G, Amanai K, Wang B, Jiang J. Interactions with Costal2 and suppressor of fused regulate nuclear translocation and activity of cubitus interruptus. Genes Dev 2000; 14:2893-2905.

24 Wang QT, Holmgren RA. Nuclear import of cubitus interruptus is regulated by hedgehog via a mechanism distinct from Ci stabilization and Ci activation. Development 2000; 127:3131-3139.

25 Wang G, Jiang J. Multiple Cos $2 / \mathrm{Ci}$ interactions regulate $\mathrm{Ci}$ subcellular localization through microtubule dependent and independent mechanisms. Dev Biol 2004; 268:493-505.

26 Zhang W, Zhao Y, Tong C, et al. Hedgehog-regulated costal2-kinase complexes control phosphorylation and proteolytic processing of cubitus interruptus. Dev Cell 2005; 8:267-278.

27 Methot N, Basler K. Suppressor of fused opposes Hedgehog signal transduction by impeding nuclear accumulation of the activator form of Cubitus interruptus. Development 2000; 
127:4001-4010

28 Ohlmeyer JT, Kalderon D. Hedgehog stimulates maturation of Cubitus interruptus into a labile transcriptional activator. Nature 1998; 396:749-753.

29 Svard J, Heby-Henricson K, Persson-Lek M, et al. Genetic elimination of suppressor of fused reveals an essential repressor function in the mammalian Hedgehog signaling pathway. Dev Cell 2006; 10:187-197.

30 Chen MH, Wilson CW, Li YJ, et al. Cilium-independent regulation of Gli protein function by Sufu in Hedgehog signaling is evolutionarily conserved. Genes Dev 2009; 23:19101928.

31 Humke EW, Dorn KV, Milenkovic L, Scott MP, Rohatgi R. The output of Hedgehog signaling is controlled by the dynamic association between suppressor of fused and the Gli proteins. Genes Dev 2010; 24:670-682.

32 Zhang Q, Zhang L, Wang B, Ou CY, Chien CT, Jiang J. A hedgehog-induced BTB protein modulates hedgehog signaling by degrading Ci/Gli transcription factor. Dev Cell 2006; 10:719-729.

33 Kent D, Bush EW, Hooper JE. Roadkill attenuates Hedgehog responses through degradation of Cubitus interruptus. Development 2006; 133:2001-2010.

34 Zhang Q, Shi Q, Chen Y, et al. Multiple Ser/Thr-rich degrons mediate the degradation of $\mathrm{Ci} / \mathrm{Gli}$ by the Cul3-HIB/SPOP E3 ubiquitin ligase. Proc Natl Acad Sci USA 2009; 106:2119121196.

35 Wang C, Pan Y, Wang B. Suppressor of fused and Spop regulate the stability, processing and function of Gli2 and Gli3 full-length activators but not their repressors. Development 2010; 137:2001-2009.

36 Wen X, Lai CK, Evangelista M, Hongo JA, de Sauvage FJ, Scales SJ. Kinetics of hedgehog-dependent full-length Gli3 accumulation in primary cilia and subsequent degradation. Mol Cell Biol 2010; 30:1910-1922.

37 Chen MH, Gao N, Kawakami T, Chuang PT. Mice deficient in the fused homolog do not exhibit phenotypes indicative of perturbed hedgehog signaling during embryonic development. Mol Cell Biol 2005; 25:7042-7053.

38 Merchant M, Evangelista M, Luoh SM, et al. Loss of the serine/threonine kinase fused results in postnatal growth defects and lethality due to progressive hydrocephalus. Mol Cell Biol 2005; 25: 7054-7068.

39 Goetz SC, Anderson KV. The primary cilium: a signalling centre during vertebrate development. Nat Rev Genet 2010; 11:331-344.

40 Huangfu D, Anderson KV. Cilia and Hedgehog responsiveness in the mouse. Proc Natl Acad Sci USA 2005; 102:1132511330 .

41 Garcia-Garcia MJ, Eggenschwiler JT, Caspary T, et al. Analysis of mouse embryonic patterning and morphogenesis by forward genetics. Proc Natl Acad Sci USA 2005; 102:59135919.

42 Corbit KC, Aanstad P, Singla V, Norman AR, Stainier DY, Reiter JF. Vertebrate Smoothened functions at the primary cilium. Nature 2005; 437:1018-1021.

43 Haycraft CJ, Banizs B, Aydin-Son Y, Zhang Q, Michaud EJ, Yoder BK. Gli2 and gli3 localize to cilia and require the intraflagellar transport protein polaris for processing and func- tion. PLoS Genet 2005; 1:e53.

44 Rohatgi R, Milenkovic L, Scott MP. Patched1 regulates hedgehog signaling at the primary cilium. Science 2007; 317:372-376.

45 Tuson M, He M, Anderson KV. Protein kinase A acts at the basal body of the primary cilium to prevent Gli2 activation and ventralization of the mouse neural tube. Development 2011; 138:4921-4930.

46 Tukachinsky H, Lopez LV, Salic A. A mechanism for vertebrate Hedgehog signaling: recruitment to cilia and dissociation of SuFu-Gli protein complexes. J Cell Biol 2010; 191:415-428.

47 Liu A, Wang B, Niswander LA. Mouse intraflagellar transport proteins regulate both the activator and repressor functions of Gli transcription factors. Development 2005; 132:3103-3111.

48 Jiang J, Struhl G. Protein kinase A and Hedgehog signalling in Drosophila limb development. Cell 1995; 80:563-572.

49 Jiang J, Struhl G. Regulation of the Hedgehog and Wingless signalling pathways by the F- box/WD40-repeat protein Slimb. Nature 1998; 391:493-496.

50 Li W, Ohlmeyer JT, Lane ME, Kalderon D. Function of protein kinase A in hedghehog signal transduction and Drosophila imaginal disc development. Cell 1995; 80:553-562.

51 Pan D, Rubin GM. cAMP-dependent protein kinase and hedgehog act antagonistically in regulating decapentaplegic transcription in Drosophila imaginal discs. Cell 1995; 80:543-552.

52 Spencer E, Jiang J, Chen ZJ. Signal-induced ubiquitination of I $\mathrm{KB} \alpha$ by the F-box protein Slimb/ $\beta$-TrCP. Genes Dev 1999; 13:284-294.

53 Winston JT, Strack P, Beer-Romero P, Chu CY, Elledge SJ, Harper JW. The SCF $\beta$-TRCP-ubiquitin ligase complex associates specifically with phosphorylated destruction motifs in $\mathrm{I} \kappa \mathrm{B} \alpha$ and $\beta$-catenin and stimulates $\mathrm{I} \kappa \mathrm{B} \alpha$ ubiquitination in vitro. Genes Dev 1999; 13:270-283.

54 Price MA, Kalderon D. Proteolysis of cubitus interruptus in Drosophila requires phosphorylation by protein kinase A. Development 1999; 126:4331-4339.

55 Wang G, Wang B, Jiang J. Protein kinase A antagonizes Hedgehog signaling by regulating both the activator and repressor forms of Cubitus interruptus. Genes Dev 1999; 13:2828-2837.

56 Jia J, Amanai K, Wang G, Tang J, Wang B, Jiang J. Shaggy/ GSK3 antagonizes Hedgehog signalling by regulating Cubitus interruptus. Nature 2002; 416:548-552.

57 Jia J, Zhang L, Zhang Q, et al. Phosphorylation by doubletime/CKIepsilon and CKI $\alpha$ targets cubitus interruptus for Slimb/ $\beta$-TRCP-mediated proteolytic processing. Dev Cell 2005; 9:819-830.

58 Price MA, Kalderon D. Proteolysis of the Hedgehog signaling effector Cubitus interruptus requires phosphorylation by glycogen synthase kinase 3 and casein kinase 1. Cell 2002; 108:823-835.

59 Smelkinson MG, Kalderon D. Processing of the Drosophila hedgehog signaling effector $\mathrm{Ci}-155$ to the repressor $\mathrm{Ci}-75$ is mediated by direct binding to the SCF component slimb. Curr Biol 2006; 16:110-116.

60 Smelkinson MG, Zhou Q, Kalderon D. Regulation of Ci- 
SCFSlimb binding, Ci proteolysis, and hedgehog pathway activity by Ci phosphorylation. Dev Cell 2007; 13:481-495.

61 Strigini M, Cohen SM. A Hedgehog activity gradient contributes to AP axial patterning of the Drosophila wing. Development 1997; 124:4697-4705.

62 Wang B, Fallon JF, Beachy PA. Hedgehog-regulated processing of Gli3 produces an anterior/posterior repressor gradient in the developing vertebrate limb. Cell 2000; 100:423-434.

63 Wang B, Li Y. Evidence for the direct involvement of $\{\beta\}$ $\mathrm{TrCP}$ in Gli3 protein processing. Proc Natl Acad Sci USA 2006; 103:33-8.

64 Pan Y, Bai CB, Joyner AL, Wang B. Sonic hedgehog signaling regulates Gli2 transcriptional activity by suppressing its processing and degradation. Mol Cell Biol 2006; 26:33653377.

65 Tempe D, Casas M, Karaz S, Blanchet-Tournier MF, Concordet JP. Multisite protein kinase A and glycogen synthase kinase $3 \beta$ phosphorylation leads to Gli3 ubiquitination by SCFßTrCP. Mol Cell Biol 2006; 26:4316-4326.

66 Bhatia N, Thiyagarajan S, Elcheva I, et al. Gli2 is targeted for ubiquitination and degradation by $\beta$-TrCP ubiquitin ligase. J Bio Chem 2006; 281:19320-19326.

67 Pan Y, Wang B. A novel protein-processing domain in Gli2 and Gli3 differentially blocks complete protein degradation by the proteasome. J Biol Chem 2007; 282:10846-10852.

68 Methot N, Basler K. Hedgehog controls limb development by regulating the activities of distinct transcriptional activator and repressor forms of Cubitus interruptus. Cell 1999; 96:819-831.

69 Wang Y, Price MA. A unique protection signal in Cubitus interruptus prevents its complete proteasomal degradation. Mol Cell Biol 2008; 28:5555-5568.

70 Marks SA, Kalderon D. Regulation of mammalian Gli proteins by Costal 2 and PKA in Drosophila reveals Hedgehog pathway conservation. Development 2011; 138:2533-2542.

71 Liu C, Li Y, Semenov M, et al. Control of $\beta$-catenin phosphorylation/degradation by a dual-kinase mechanism. Cell 2002; 108:837-847.

72 Zhang L, Jia J, Wang B, Amanai K, Wharton KA, Jr, Jiang J. Regulation of wingless signaling by the CKI family in Drosophila limb development. Dev Biol 2006; 299:221-237.

73 MacDonald BT, Tamai K, He X. Wnt/ $\beta$-catenin signaling: components, mechanisms, and diseases. Dev cell 2009; 17:926.

74 Kise Y, Morinaka A, Teglund S, Miki H. Sufu recruits GSK3 $\beta$ for efficient processing of Gli3. Biochem Biophys Res Commun 2009; 387:569-574.

75 Cheung HO, Zhang X, Ribeiro A, et al. The kinesin protein Kif7 is a critical regulator of Gli transcription factors in mammalian hedgehog signaling. Sci Signal 2009; 2:ra29.

76 Endoh-Yamagami S, Evangelista M, Wilson D, et al. The mammalian Cos2 homolog Kif7 plays an essential role in modulating Hh signal transduction during development. Curr Biol 2009; 19:1320-1326.

77 Liem KF Jr, He M, Ocbina PJ, Anderson KV. Mouse $\mathrm{Kif7} / \mathrm{Costa} 2$ is a cilia-associated protein that regulates Sonic hedgehog signaling. Proc Natl Acad Sci USA 2009; 106:13377-13382.

78 Xie J, Murone M, Luoh S-M, et al. Activating Smoothened mutations in sporadic basal-cell carcinoma. Nature 1998; 391:90-92.

79 Rubin LL, de Sauvage FJ. Targeting the Hedgehog pathway in cancer. Nat Rev Drug Discov 2006; 5:1026-1033.

80 Ohlmeyer JT, Kalderon D. Dual pathways for induction of wingless expression by protein kinase A and Hedgehog in Drosophila embryos. Genes Dev 1997; 11:2250-2258.

81 Jia J, Tong C, Wang B, Luo L, Jiang J. Hedgehog Signalling activity of Smoothened requires phosphorylation by protein kinase A and casein kinase I. Nature 2004; 432:1045-1050.

82 Zeng X, Tamai K, Doble B, et al. A dual-kinase mechanism for Wnt co-receptor phosphorylation and activation. Nature 2005; 438:873-877.

83 Apionishev S, Katanayeva NM, Marks SA, Kalderon D, Tomlinson A. Drosophila Smoothened phosphorylation sites essential for Hedgehog signal transduction. Nat Cell Biol 2005; 7:86-92.

84 Zhang C, Williams EH, Guo Y, Lum L, Beachy PA. Extensive phosphorylation of Smoothened in Hedgehog pathway activation. Proc Natl Acad Sci USA 2004; 101:17900-17907.

85 Fan J, Liu Y, Jia J. Hh-induced Smoothened conformational switch is mediated by differential phosphorylation at its Cterminal tail in a dose- and position-dependent manner. Dev Biol 2012; 366:172-184.

86 Xia R, Jia H, Fan J, Liu Y, Jia J. USP8 promotes Smoothened signaling by preventing its ubiquitination and changing its subcellular localization.PloS Biol 2012; 10:e1001238.

87 Li S, Chen Y, Shi Q, Yue T, Wang B, Jiang J. Hedgehogregulated ubiquitination controls Smoothened trafficking and cell surface expression in Drosophila. PLoS Biol 2012; 10:e1001239.

88 Molnar C, Ruiz-Gomez A, Martin M, Rojo-Berciano S, Mayor F, de Celis JF. Role of the Drosophila non-visual ssarrestin kurtz in hedgehog signalling. PLoS Genet 2011; 7:e1001335.

89 Mukai A, Yamamoto-Hino M, Awano W, Watanabe W, Komada $\mathrm{M}$, Goto S. Balanced ubiquitylation and deubiquitylation of frizzled regulate cellular responsiveness to $\mathrm{Wg} / \mathrm{Wnt}$. EMBO J 2010; 29:2114-2125.

90 Zhao Y, Tong C, Jiang J. Hedgehog regulates Smoothened activity by inducing a conformational switch. Nature 2007; 450:252-258.

91 Jia H, Liu Y, Xia R, et al. Casein kinase 2 promotes Hedgehog signaling by regulating both Smoothened and Cubitus interruptus. J Biol Chem 2010; 285:37218-37226.

92 Chen Y, Li S, Tong C, et al. G protein-coupled receptor kinase 2 promotes high-level Hedgehog signaling by regulating the active state of Smo through kinase-dependent and kinaseindependent mechanisms in Drosophila. Genes Dev 2010; 24:2054-2067.

93 Chen W, Ren XR, Nelson CD, et al. Activity-dependent internalization of Smoothened mediated by $\beta$-arrestin 2 and GRK2. Science 2004; 306:2257-2260.

94 Meloni AR, Fralish GB, Kelly P, et al. Smoothened signal transduction is promoted by $\mathrm{G}$ protein-coupled receptor kinase 2. Mol Cell Biol 2006; 26:7550-7560.

95 Molnar C, Holguin H, Mayor F Jr, Ruiz-Gomez A, de Celis JF. The G protein-coupled receptor regulatory kinase GPRK2 participates in Hedgehog signaling in Drosophila. Proc Natl 
Acad Sci USA 2007; 104:7963-7968.

96 Zhang S, Wang Y, Mao JH, et al. Inhibition of CK2 $\alpha$ downregulates Hedgehog/Gli signaling leading to a reduction of a stem-like side population in human lung cancer cells. PloS One 2012; 7:e38996.

97 Cheng S, Maier D, Neubueser D, Hipfner DR. Regulation of Smoothened by Drosophila G-protein-coupled receptor kinases. Dev Biol 2010; 337:99-109.

98 Huangfu D, Anderson KV. Signaling from Smo to $\mathrm{Ci} / \mathrm{Gli}$ : conservation and divergence of Hedgehog pathways from Drosophila to vertebrates. Development 2006; 133:3-14.

99 Varjosalo M, Li SP, Taipale J. Divergence of hedgehog signal transduction mechanism between Drosophila and mammals. Dev Cell 2006; 10:177-186.

100 Chen Y, Sasai N, Ma G, et al. Sonic Hedgehog dependent phosphorylation by CK1a and GRK2 is required for ciliary accumulation and activation of smoothened. PLoS Biology 2011; 9:e1001083.

101 Evangelista M, Lim TY, Lee J, et al. Kinome siRNA screen identifies regulators of ciliogenesis and hedgehog signal transduction. Sci Signal 2008; 1:ra7.

102 Kovacs JJ, Whalen EJ, Liu R, et al. $\beta$-arrestin-mediated localization of Smoothened to the primary cilium. Science 2008; 320:1777-1781.

103 Rohatgi R, Milenkovic L, Corcoran RB, Scott MP. Hedgehog signal transduction by Smoothened: pharmacologic evidence for a 2-step activation process. Proc Natl Acad Sci USA 2009; 106:3196-3201.

104 Wang Y, Zhou Z, Walsh CT, McMahon AP. Selective translocation of intracellular Smoothened to the primary cilium in response to Hedgehog pathway modulation. Proc Natl Acad Sci USA 2009; 106:2623-2628.

105 Wilson CW, Chen MH, Chuang PT. Smoothened adopts multiple active and inactive conformations capable of trafficking to the primary cilium. PLoS One 2009; 4:e5182.

106 DeCamp DL, Thompson TM, de Sauvage FJ, Lerner MR. Smoothened activates Gai-mediated signaling in frog melanophores. J Biol Chem 2000; 275:26322-26327.

107 Riobo NA, Saucy B, Dilizio C, Manning DR. Activation of heterotrimeric G proteins by Smoothened. Proc Natl Acad Sci USA 2006; 103:12607-12612.

108 Ogden SK, Fei DL, Schilling NS, Ahmed YF, Hwa J, Robbins DJ. G protein Ga(i) functions immediately downstream of Smoothened in Hedgehog signalling. Nature 2008; 456:967-970

109 Low WC, Wang C, Pan Y, Huang XY, Chen JK, Wang B. The decoupling of Smoothened from Gai proteins has little effect on Gli3 protein processing and Hedgehog-regulated chick neural tube patterning. Dev Biol 2008; 321: 188-196.

110 Wilson CW, Chuang PT. Mechanism and evolution of cytosolic Hedgehog signal transduction. Development 2010; 137:2079-2094.

111 Jia J, Tong C, Jiang J. Smoothened transduces Hedgehog signal by physically interacting with Costal2/Fused complex through its C-terminal tail. Genes Dev 2003; 17:2709-2720.

112 Lum L, Zhang C, Oh S, et al. Hedgehog signal transduction via Smoothened association with a cytoplasmic complex scaffolded by the atypical kinesin, Costal-2. Mol Cell 2003; 12:1261-1274.
113 Ogden SK, Ascano M Jr, Stegman MA, Suber LM, Hooper JE, Robbins DJ. Identification of a functional interaction between the transmembrane protein Smoothened and the kinesin-related protein Costal2. Curr Biol 2003; 13:19982003.

114 Ruel L, Rodriguez R, Gallet A, Lavenant-Staccini L, Therond PP. Stability and association of Smoothened, Costal2 and Fused with cubitus interruptus are regulated by Hedgehog. Nat Cell Biol 2003; 5:907-913.

115 Hooper JE. Smoothened translates Hedgehog levels into distinct responses. Development 2003; 130:3951-3963.

116 Shi Q, Li S, Jia J, Jiang J. The Hedgehog-induced Smoothened conformational switch assembles a signaling complex that activates Fused by promoting its dimerization and phosphorylation. Development 2011; 138:4219-4231.

117 Zhang Y, Mao F, Lu Y, Wu W, Zhang L, Zhao Y. Transduction of the Hedgehog signal through the dimerization of Fused and the nuclear translocation of Cubitus interruptus. Cell research 2011; 21:1436-1451.

118 Jia H, Liu Y, Yan W, Jia J. PP4 and PP2A regulate Hedgehog signaling by controlling Smo and Ci phosphorylation. Development 2009; 136:307-316.

119 Liu Y, Cao X, Jiang J, Jia J. Fused-Costal2 protein complex regulates Hedgehog-induced Smo phosphorylation and cellsurface accumulation. Genes Dev 2007; 21:1949-1963.

120 Claret S, Sanial M, Plessis A. Evidence for a novel feedback loop in the Hedgehog pathway involving Smoothened and Fused. Curr Biol 2007; 17:1326-1333.

121 Nybakken KE, Turck CW, Robbins DJ, Bishop JM. Hedgehog-stimulated phosphorylation of the kinesin-related protein Costal2 is mediated by the serine/threonine kinase fused. $J$ Biol Chem 2002; 277:24638-24647.

122 Ruel L, Gallet A, Raisin S, et al. Phosphorylation of the atypical kinesin Costal2 by the kinase Fused induces the partial disassembly of the Smoothened-Fused-Costal2-Cubitus interruptus complex in Hedgehog signalling. Development 2007; 134:3677-3689.

123 Ranieri N, Ruel L, Gallet A, Raisin S, Therond PP. Distinct phosphorylations on kinesin costal-2 mediate differential hedgehog signaling strength. Developmental cell 2012; 22:279-294.

124 Alves G, Limbourg-Bouchon B, Tricoire H, Brissard-Zahraoui J, Lamour-Isnard C, Busson D. Modulation of Hedgehog target gene expression by the Fused serine- threonine kinase in wing imaginal discs. Mech Dev 1998; 78:17-31.

125 Wolff C, Roy S, Ingham PW. Multiple muscle cell identities induced by distinct levels and timing of hedgehog activity in the zebrafish embryo. Curr Biol 2003; 13:1169-1181.

126 Monnier V, Dussillol F, Alves G, Lamour-Isnard C, Plessis A. Suppressor of Fused links fused and Cubitus interruptus on the hedgehog signalling pathway. Curr Biol 1998; 8:583-586.

127 Ascano M Jr, Robbins DJ. An intramolecular association between two domains of the protein kinase Fused is necessary for Hedgehog signaling. Mol Cell Biol 2004; 24:1039710405.

128 Therond PP, Knight JD, Kornberg TB, Bishop JM. Phosphorylation of the Fused protein kinase in response to signaling from hedgehog. Proc Natl Acad Sci USA 1996; 93:42244228 . 
129 Zhou Q, Kalderon D. Hedgehog activates Fused through phosphorylation to elicit a full spectrum of pathway responses. Dev Cell 2011; 20:802-814.

130 Pike AC, Rellos P, Niesen FH, et al. Activation segment dimerization: a mechanism for kinase autophosphorylation of non-consensus sites. EMBO J 2008; 27:704-714.

131 Raisin S, Ruel L, Ranieri N, Staccini-Lavenant L, Therond PP. Dynamic phosphorylation of the kinesin Costal-2 in vivo reveals requirement of Fused kinase activity for all levels of hedgehog signalling. Dev Biol 2010; 344:119-128.

132 Cooper AF, Yu KP, Brueckner M, et al. Cardiac and CNS defects in a mouse with targeted disruption of suppressor of Fused. Development 2005; 132:4407-4417.

133 Barnfield PC, Zhang X, Thanabalasingham V, Yoshida M, Hui CC. Negative regulation of Gli1 and Gli2 activator function by Suppressor of Fused through multiple mechanisms. Differentiation 2005; 73:397-405.

134 Ding Q, Fukami S, Meng X, et al. Mouse suppressor of fused is a negative regulator of sonic hedgehog signaling and alters the subcellular distribution of Gli1. Curr Biol 1999; 9:11191122.

135 Cheng SY, Bishop JM. Suppressor of Fused represses Glimediated transcription by recruiting the SAP18-mSin 3 corepressor complex. Proc Natl Acad Sci USA 2002; 99:54425447.

136 Merchant M, Vajdos FF, Ultsch M, et al. Suppressor of fused regulates Gli activity through a dual binding mechanism. Mol Cell Biol 2004; 24:8627-8641.

137 Maloverjan A, Piirsoo M, Michelson P, Kogerman P, Osterlund T. Identification of a novel serine/threonine kinase ULK 3 as a positive regulator of Hedgehog pathway. Exp Cell Res 2010; 316:627-637.

138 Yue S, Chen Y, Cheng SY. Hedgehog signaling promotes the degradation of tumor suppressor Sufu through the ubiquitinproteasome pathway. Oncogene 2009; 28:492-499.

139 Chen Y, Yue S, Xie L, Pu XH, Jin T, Cheng SY. Dual Phosphorylation of suppressor of fused (Sufu) by PKA and GSK $3 \beta$ regulates its stability and localization in the primary cilium. J Biol Chem 2011; 286:13502-13511.

140 Hsu SH, Zhang X, Yu C, et al. Kif7 promotes hedgehog sig- naling in growth plate chondrocytes by restricting the inhibitory function of Sufu. Development 2011; 138:3791-3801.

141 Yang C, Chen Y, Jiang J. Smoothened transduces Hedgehog signal by forming a complex with Evc/Evc2. Cell Res 2012; 22:1593-1604.

142 Ruiz-Perez VL, Blair HJ, Rodriguez-Andres ME, et al. Evc is a positive mediator of Ihh-regulated bone growth that localises at the base of chondrocyte cilia. Development 2007; 134:2903-2912.

143 Blair HJ, Tompson S, Liu YN, et al. Evc2 is a positive modulator of Hedgehog signalling that interacts with Evc at the cilia membrane and is also found in the nucleus. BMC Biol 2011; 9:14.

144 Dorn KV, Hughes CE, Rohatgi R. A Smoothened-Evc2 complex transduces the Hedgehog signal at primary cilia. Dev Cell 2012; 23:823-835.

145 Stuckemann T, Wegleiter T, Stefan E, et al. Zebrafish Cxcr4a determines the proliferative response to Hedgehog signalling. Development 2012; 139:2711-2720.

146 Cheng S, Maier D, Hipfner DR. Drosophila G-protein-coupled receptor kinase 2 regulates cAMP-dependent Hedgehog signaling. Development 2012; 139:85-94.

147 Taipale J, Cooper MK, Maiti T, Beachy PA. Patched acts catalytically to suppress the activity of Smoothened. Nature 2002; 418:892-897.

148 Nachtergaele S, Mydock LK, Krishnan K, et al. Oxysterols are allosteric activators of the oncoprotein Smoothened. Nat Chem Biol 2012; 8:211-220.

149 Yavari A, Nagaraj R, Owusu-Ansah E, et al. Role of lipid metabolism in smoothened derepression in hedgehog signaling. Dev Cell 2010; 19:54-65.

150 Wang Y, Ding Q, Yen CJ, et al. The crosstalk of mTOR/S6K1 and Hedgehog pathways. Cancer cell 2012; 21:374-387.

This work is licensed under the Creative Commons Attribution-NonCommercial-No Derivative Works 3.0 Unported License. To view a copy of this license, visit http:// creativecommons.org/licenses/by-nc-nd/3.0 